ISSN 1112-9867

Available online at

http://www.jfas.info

\title{
INVERSE CORRECTION OF FOURIER TRANSFORMS FOR ONE-DIMENSIONAL STRONGLY SCATTERING APERIODIC LATTICES
}

\author{
Ying-Fei Hsin
}

Institute of Medical Sciences, Tzu Chi University, Hualien, 97004, Taiwan

Received: 14 December 2015 / Accepted: 29 April 2016 / Published online: 01 May 2016

\begin{abstract}
The accuracy of the Fourier transform (FT), advantageous for the aperiodic lattice (AL) design, is significantly improved for strongly scattering periodic lattices (PLs) and ALs. The approach is to inversely obtain corrected parameters from an accurate transfer matrix method for the FT. We establish a corrected FT in order to improve the spectral inaccuracy of strongly scattering PLs by redefining wave numbers and reflective intensity. We further correct the FT for strongly scattering ALs by implementing improvements applied to strongly scattering PLs and then making detailed wave number adjustments in the main band spectral region. Silicon lattice simulations are presented.
\end{abstract}

Keywords: Fourier transform algorithm; aperiodic lattice; strong scattering; Bragg resonant behavior; transfer matrix method; Riccati differential equation.

Author Correspondence, e-mail: 104353112@gms.tcu.edu.tw

doi: http://dx.doi.org/10.4314/jfas.v8i2.27

\section{INTRODUCTION}

The renormalization technique, widely used in inverse scattering problems, is an interesting issue when applied to photonic bandgap structures for photonic integrated circuits (PICs) and dense wavelength-division-multiplexing (DWDM) systems [1,2]. The nonlinear Riccati 
differential equation technique, from which the Fourier transform (FT) formula can be derived, is commonly used for reconstruction in scattering conditions $[3,4]$. Therefore, the relationship between the reflection spectrum and the refractive index for a scattering medium is established by implementing the fast FT or inverse FT formula.

Recently, the aperiodic lattice (AL) was developed as a novel design for the control of light localization and transport properties utilizing the lack of translational symmetry $[5,6]$. The FT algorithm is particularly useful for inversely calculating an aperiodic grating design from a target reflective spectrum. Some algorithms based on the FT algorithm, such as the Fourier (k-) space design approach, have been investigated for this purpose [7]. Unfortunately, the FT formula suffers from inaccuracy as the scattering strength increases due to phase-accumulation error and predicts a narrower photonic bandgap. An improved FT formula has been investigated for certain cases [8,9]. This improved method uses the Debye-Waller approximation in order to model Bragg resonant behavior of the refractive index and coupling coefficient. However, examples of improved FT algorithms in the literature may overly simplify the resonant refractive index, particularly when Bragg resonant behavior occurs in an aperiodic lattice. Other improvements involve combining the Riccati differential equation with optimization algorithms. Nevertheless, slow iterative schemes for optimization hinder the fast and effective feature of FT calculations [10,11].

As it is widely used in periodic lattice design theory and is particularly useful in aperiodic lattice design $[12,13]$, the accuracy of the FT algorithm under strong scattering conditions is the focus of this paper. We propose an inverse correction approach for the inaccurate FT algorithm in strongly scattering lattices. The proposed scheme employs a conventional Transfer Matrix (TM) method, an accurate algorithm for plotting a reflective spectrum from a refractive index profile, to inversely obtain corrected functions of the inaccurate FT formula. Once a corrected FT formula is achieved, the inverse FT formula is also corrected. We first investigate this corrected approach for the FT of periodic strongly scattering lattices. Corrections to the FT for aperiodic lattices are achieved using our improved approach for periodic lattices, but by making further corrections in the main band spectral region. Several simulations of silicon periodic and aperiodic lattices are presented. 


\section{TRANSFER MATRIX METHOD FOR MODELING MULTILAYER FILTERS}

The transfer matrix method (TMM) for modeling the transmission and reflection spectra of multilayer filters was established assuming layer boundary conditions [14,15]. Fig. 1 shows forward and backward traveling fields in several layers.

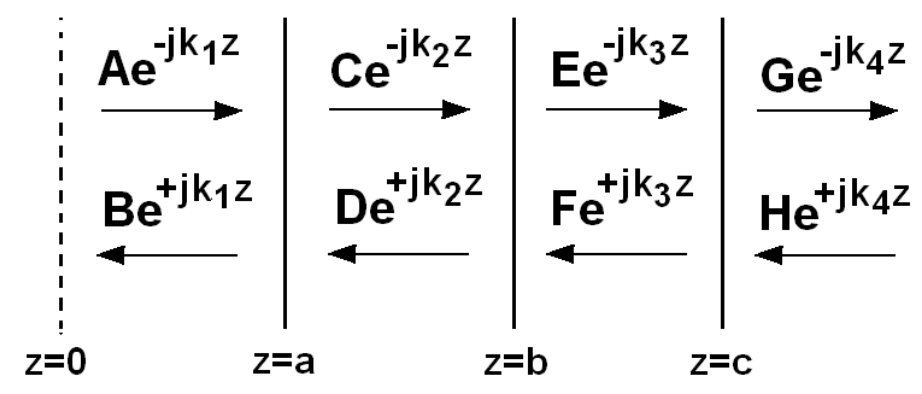

Fig.1. The forward and backward traveling fields in a multilayer filter

The field amplitudes and their derivatives are continuous when the field passes through each layer boundary. Define $M_{n}(z)$ to be

$M_{n}(z)=\left[\begin{array}{cc}\exp \left(-j k_{n} z\right) & \exp \left(j k_{n} z\right) \\ -j k_{n} \exp \left(-j k_{n} z\right) & j k_{n} \exp \left(j k_{n} z\right)\end{array}\right]$.

Therefore, at the first boundary $(\mathrm{z}=\mathrm{a})$,

$M_{1}(a)\left[\begin{array}{l}A \\ B\end{array}\right]=M_{2}(a)\left[\begin{array}{l}C \\ D\end{array}\right] \Rightarrow M_{2}^{-1}(a) M_{1}(a)\left[\begin{array}{l}A \\ B\end{array}\right]=\left[\begin{array}{l}C \\ D\end{array}\right]$.

Similarly, at the second boundary $(\mathrm{z}=\mathrm{b})$

$M_{2}(b)\left[\begin{array}{l}C \\ D\end{array}\right]=M_{3}(b)\left[\begin{array}{l}E \\ F\end{array}\right] \Rightarrow M_{3}^{-1}(b) M_{2}(b)\left[\begin{array}{l}C \\ D\end{array}\right]=\left[\begin{array}{l}E \\ F\end{array}\right]$,

which results in

$M_{3}^{-1}(b) M_{2}(b) M_{2}^{-1}(a) M_{1}(a)\left[\begin{array}{l}A \\ B\end{array}\right]=\left[\begin{array}{l}E \\ F\end{array}\right]$.

For a structure composed of $\mathrm{N}$ layers, we define $Y$ and $Z$ to be field amplitudes in the last layer, equation (4) can then be written as

$$
M_{N}^{-1}\left(z_{N}\right) M_{N-1}\left(z_{N}\right) \ldots \ldots . . . M_{3}^{-1}(b) M_{2}(b) M_{2}^{-1}(a) M_{1}(a)\left[\begin{array}{l}
A \\
B
\end{array}\right]=\left[\begin{array}{l}
Y \\
Z
\end{array}\right]
$$


$\Rightarrow T\left[\begin{array}{l}A \\ B\end{array}\right]=\left[\begin{array}{l}Y \\ Z\end{array}\right]$,

where $T$ is the product of all the individual matrices. The boundary condition is $\mathrm{z}=0$ since there is no wave reflected back in the last layer. Assuming the amplitude of the incident field is 1 , equation (5) becomes

$T\left[\begin{array}{l}1 \\ r\end{array}\right]=\left[\begin{array}{l}t \\ 0\end{array}\right]$,

Where $\mathrm{t}$ and $\mathrm{r}$ are the transmission and reflection coefficient, respectively. Thus, we can derive equations at a particular wavelength:

$r=-T_{21} / T_{22}, \quad t=T_{11}+T_{12} r$.

Transmission and reflection spectra are then obtained by performing calculations under different wavelength conditions.

\section{ACCURATE FT IN WEAKLY SCATTERING PERIODIC LATTICES}

The Riccati equation derived by Jaggard and Kim [3] can be expressed as

$\frac{d r\left(z, k_{0}\right)}{d z}=\frac{1}{2}\left[1-r^{2}\left(z, k_{0}\right)\right] \frac{d}{d z}\{\ln [k(z)]\}-2 i k(z) r\left(z, k_{0}\right)$,

where $k(z)=\left\{\begin{array}{ll}k_{0}, & z<0 \\ k_{0} n(z), & z \geq 0\end{array}\right.$,

$k(z)$ is the local wave number, and $k_{0}$ is its free-space value. From the Riccati equation, the complex reflection coefficient $r\left(k_{0}\right)$ at all wave numbers $k_{0}$ is found from the knowledge of the refractive index. Dividing Eq. (8) by a factor $\left[1-r^{2}\left(z, k_{0}\right)\right]$ yields the equation

$\frac{1}{\left[1-r^{2}\left(z, k_{0}\right)\right]} \frac{d r\left(z, k_{0}\right)}{d z}=\frac{1}{2} \frac{d}{d z}\{\ln [k(z)]\}-\frac{2 i k(z) r\left(z, k_{0}\right)}{\left[1-r^{2}\left(z, k_{0}\right)\right]}$.

Defining a new variable $\hat{r}\left(z, k_{0}\right) \equiv \tanh ^{-1}\left[r\left(z, k_{0}\right)\right] \approx r\left(z, k_{0}\right)\left[1-r^{2}\left(z, k_{0}\right)\right]^{-1}$ and inserting it into Eq. (10) yields

$\frac{d \hat{r}\left(z, k_{0}\right)}{d z}=\frac{1}{2} \frac{d}{d z}\{\ln [n(z)]\}-2 i k_{0} n(z) \hat{r}\left(z, k_{0}\right)$.

Eq. (11) becomes a linear equation in the new variable $\hat{r}\left(z, k_{0}\right)$, with solution 
$\hat{r}\left(k_{0}\right)=-\frac{1}{2} \int_{0}^{\infty} \frac{d}{d z^{\prime \prime}}\left\{\ln \left[k\left(z^{\prime \prime}\right)\right]\right\} \exp \left(2 i k_{0} \int_{0}^{z^{\prime \prime}} n(z) d z\right) d z^{\prime \prime}$.

Therefore, restoring $r\left(k_{0}\right)$ from $\hat{r}\left(k_{0}\right) \equiv \tanh ^{-1}\left[r\left(k_{0}\right)\right]$ to yield the equation

$r\left(k_{0}\right)=\tanh \left|-\frac{1}{2} \int_{0}^{\infty} \frac{d}{d z^{\prime \prime}}\left\{\ln \left[k\left(z^{\prime \prime}\right)\right]\right\} \exp \left(2 i k_{0} \int_{0}^{z^{\prime \prime}} n(z) d z\right) d z^{\prime \prime}\right|$.

Rewriting Eq. (13) by replacing $n=\sqrt{\varepsilon}$, we get

$r\left(k_{0}\right)=\tanh \left|-\frac{1}{4} \int_{0}^{\infty} \frac{1}{\varepsilon(x)} \frac{d \varepsilon(x)}{d x} \exp \left(2 i k_{0} \int_{0}^{x} n(z) d z\right) d x\right|$.

An acceptable approximation under weakly scattering lattice conditions is to take $\varepsilon(x)$ outside of the integral as $\bar{n}^{2}$. Accordingly,

$r\left(k_{0}\right)=\tanh |\underbrace{-\frac{1}{4 \bar{n}^{2}} \int_{0}^{\infty} \frac{d \varepsilon(x)}{d x}}_{\text {AmplitudeTerm }} \underbrace{\exp \left(2 i k_{0} \int_{0}^{x} n(z) d z\right) d x}_{\text {PhaseTerm }}|$.

This equation is the Fourier transform implemented through the following sections, and Eq. (15) is accurate in weakly scattering photonic lattices but inaccurate under strong scattering conditions.

\section{CORRECTION OF FOURIER TRANSFORM FOR PERIODIC LATTICES WITH A HIGH-CONTRAST REFRACTIVE INDEX}

\subsection{Principle for periodic lattices}

Figure 2 shows a typical inaccurate reflective spectrum of a FT for strongly scattering lattices, calculated by equation (15). Compared with the TMM, this inaccurate FT shows a narrower bandwidth, wider sub-bandwidths on both sides, and a weaker reflectivity in some cases. We propose to improve the FT using Eq. (15) by using an accurate TMM as the target spectrum to inversely adjust the amplitude term by adding a Weightfactor and redefining the wave number $k(z)$ in the phase term. Accordingly, we first define $\mathrm{f} 1, \mathrm{f} 2, \ldots$ and $\mathrm{F} 1, \mathrm{~F} 2, \ldots$ in order to express the minimum points of the left and right sides of the original FT profile. We also 
define $\mathrm{t} 1, \mathrm{t} 2, \ldots$ and $\mathrm{T} 1, \mathrm{~T} 2, \ldots$ on the left and right sides for the TMM profile (see Figure 2 ).

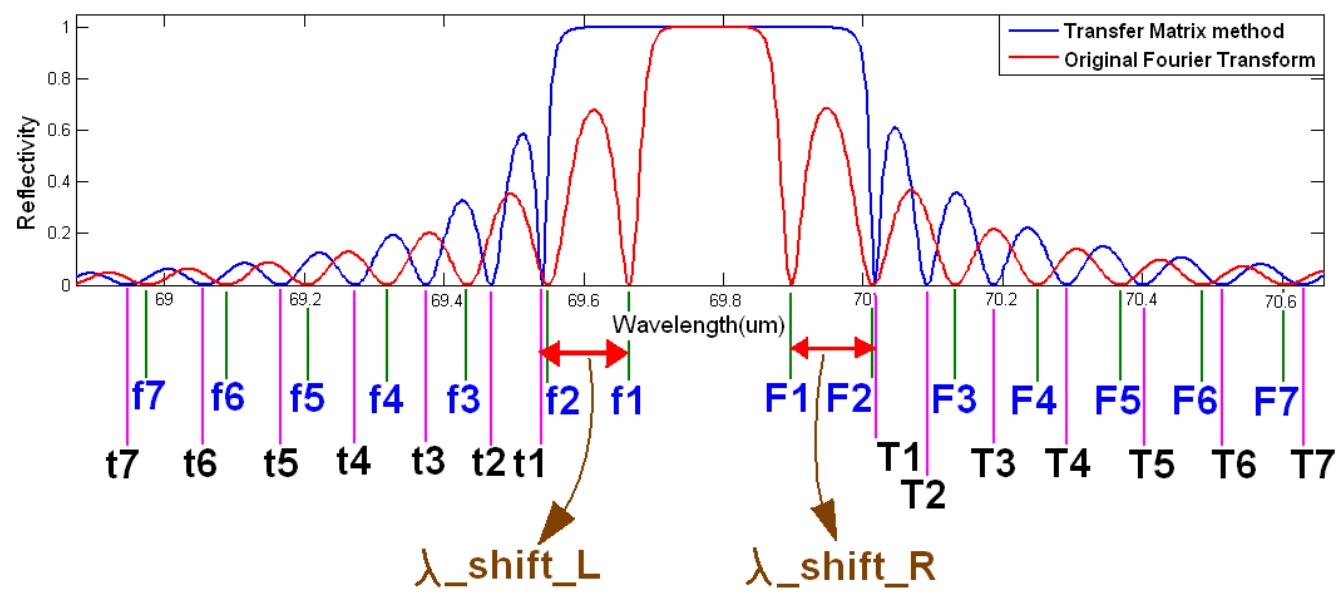

Fig.2. A typical inaccurate spectrum from the original FT formula

After identifying the minimum points, we establish

$\lambda_{-}$shift_ $R=T 1-F 1$,

$\lambda_{-}$shift_L $L=f 1-t 1$,

$A D J_{-} R(\lambda)=[F(n)-F(n-1)] /[T(n)-T(n-1)]$,

$A D J_{-} L(\lambda)=[f(n)-f(n+1)] /[t(n)-t(n+1)]$.

Eqs. (16) and (17) indicate how the wave number in each spectral point should be adjusted.

The purpose of $\lambda_{-}$shift $t_{-} R$ and $\lambda_{-}$shift $L_{-}$is to expand the bandwidth; $A D J_{-} R$ and $A D J_{-} L$ determine the degree of width adjustment of the sub-bandwidths on both sides.

Here, $k(z)=2 \pi n / \lambda$ is one of the target parameters to adjust in the FT.

Hence, the original FT is rewritten as

For $\lambda>\left(\lambda_{-}\right.$shift_ $\left.R+\lambda_{\text {Bragg }}\right)$,

$r\left(\lambda_{R}\right)=\tanh \mid-\frac{1}{4 \bar{n}^{2}} \int_{0}^{\infty} \frac{d \varepsilon(x)}{d x} \times$ Weightfactor $\times \exp \left(2 i\left(\frac{2 \pi}{\lambda_{R}^{a}}\right) \int_{0}^{x} n(z) d z\right) d x \mid ;$

For $\left(\lambda_{\text {Bragg }}-\lambda_{-}\right.$shift_L $\left.L\right) \leq \lambda \leq\left(\lambda_{\text {Bragg }}+\lambda_{-}\right.$shift_ $\left.R\right)$,

$r(\lambda)=r\left(\lambda_{\text {Bragg }}\right)$ 
For $\lambda<\left(\lambda_{\text {Bragg }}-\lambda_{-}\right.$shift_L $\left.L\right)$,

$$
r\left(\lambda_{L}\right)=\tanh \mid-\frac{1}{4 \bar{n}^{2}} \int_{0}^{\infty} \frac{d \varepsilon(x)}{d x} \times \text { Weightfactor } \times \exp \left(2 i\left(\frac{2 \pi}{\lambda_{L}^{a}}\right) \int_{0}^{x} n(z) d z\right) d x \mid .
$$

Here, $\lambda_{\text {Bragg }}$ is the Bragg wavelength, and

$$
\begin{aligned}
& \lambda_{R}^{a}=\left(\lambda_{R}-\lambda_{R-1}\right) \times A D J_{-} R\left(\lambda_{R}\right)+\lambda_{R-1}^{a}, \\
& \lambda_{L}^{a}=\lambda_{L+1}^{a}-\left(\lambda_{L+1}-\lambda_{L}\right) \times A D J_{-} L\left(\lambda_{L}\right) .
\end{aligned}
$$

Eq. (21) reveals the adjusted magnitude of the wavelength (wave number) in each spectral point. The initial values of $\lambda_{R-1}$ and $\lambda_{R-1}^{a}$ are $\left(\lambda_{-}\right.$shift $\left.f_{-} R+\lambda_{\text {Bragg }}\right)$. The initial values of $\lambda_{L+1}$ and $\lambda_{L+1}^{a}$ are $\left(\lambda_{\text {Bragg }}-\lambda_{-}\right.$shift $\left.\_L\right)$. In addition, $\left(\lambda_{R}-\lambda_{R-1}\right)$ and $\left(\lambda_{L+1}-\lambda_{L}\right)$ indicate the resolution distance of the wavelengths. The Weightfactor parameter adjusts the reflectivity. Here, $\lambda_{-}$shift $L_{-}, \lambda_{-}$shift ${ }_{-} R$, Weightfactor, $A D J_{-} R$ and $A D J_{-} L$ are obtained according to the accurate spectrum of the TMM.

\subsection{Simulation results}

We verify our corrected FT using examples of silicon periodic gratings under strongly scattering conditions for DWDM systems. We used the MATLAB environment for all calculations. The first example is shown in Fig. 3(a). The total length of the periodic grating is $11.428 \mu \mathrm{m}$ with 49 period numbers and $233.22 \mathrm{~nm}$ for the periodic pitch width. Within each period, the grating is composed of two types of refractive index: 1.86 corresponding to a width of $29.9 \mathrm{~nm}$ and 3.47 corresponding to a width of $203.32 \mathrm{~nm}$. The difference between the two refractive indices $\Delta n$ is 1.61 . The central Bragg wavelength of this filter is $1543 \mathrm{~nm}$. After calculating through the TMM and the original FT in order to inversely establish the corrected FT, the reflection profile of the spectrum is successfully adjusted by increasing Weightfactor to 1.4475 and setting $24.384 \mathrm{~nm}$ and $70.715 \mathrm{~nm}$ for $\lambda_{-}$shift $L_{-}$and $\lambda_{-}$shift_ $R$, respectively. $A D J_{-} R$ and $A D J_{-} L$ are also provided to readjust new wave numbers. In the profile of the corrected FT, the maximum points of the bandwidth and sub-bandwidths on 
both sides meet the maximum points of that in the profile of the TMM, showing that the designated wavelengths for filtering are correctly presented after correction.
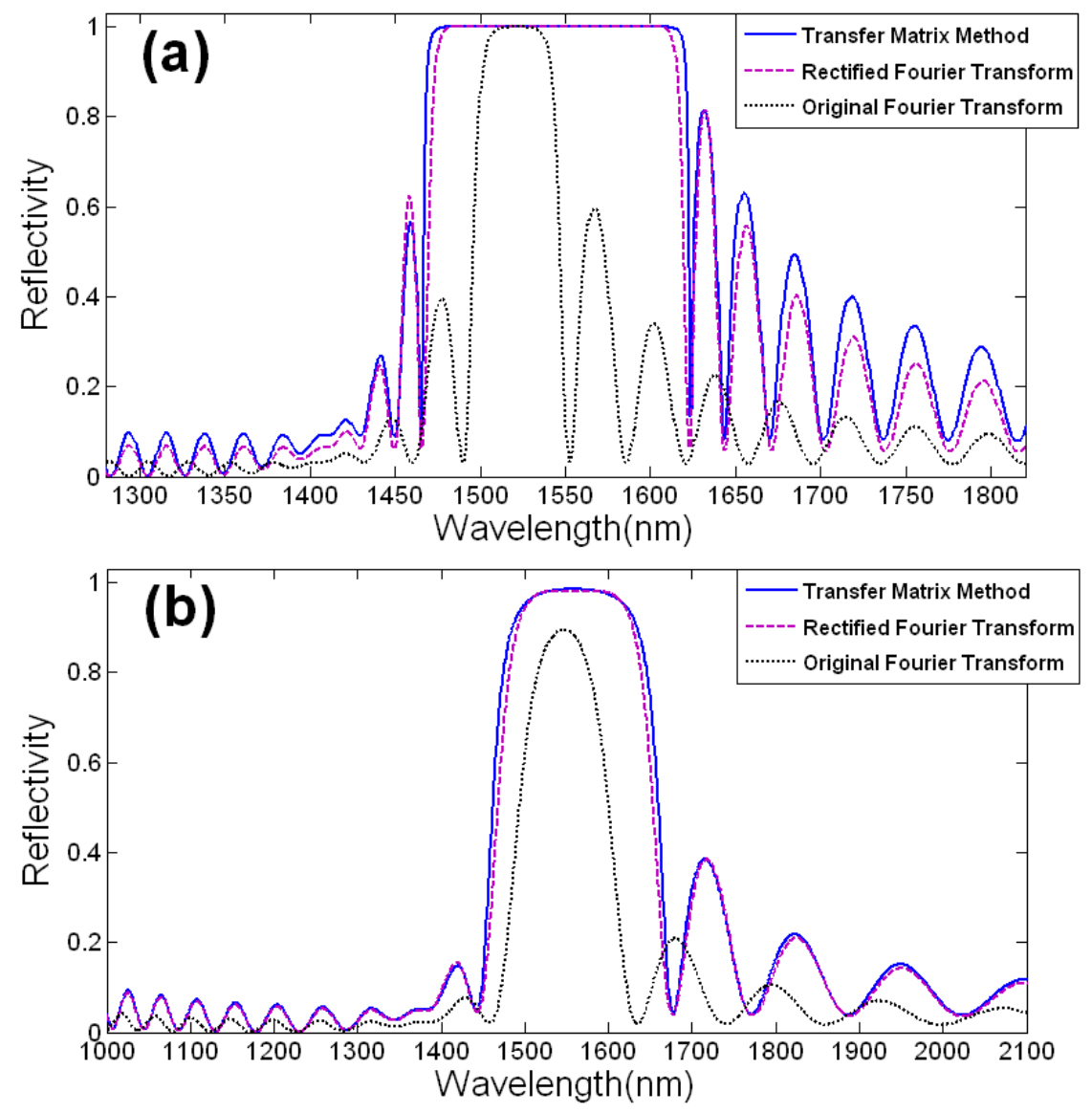

Fig.3. A comparison of the corrected FT with the TMM and the original FT. (a) Periodic grating with 49 periods. (b) Periodic grating with 18 periods

In the second example, a periodic grating with 18 periods and a $235.6 \mathrm{~nm}$ period width is presented. The total length of this grating is $4.2408 \mu \mathrm{m}$. Each period consists of two refractive indices: 3.47 and 2.2. For each period, the refractive index of 3.47 corresponds to a width of $197.6 \mathrm{~nm}$ and 2.2 corresponds to a width of $38 \mathrm{~nm}$. Here, $\Delta n$ is then 1.27. The central Bragg wavelength is located at $1554 \mathrm{~nm}$. By calculating TMM and the original FT, the corrected parameters are found as 1.4715 for Weightfactor, 15.367 in $\lambda_{-}$shift_ $L, 42.485$ in $\lambda_{-}$shift $R_{-} R$, and the database of $A D J_{-} R$ and $A D J_{-} L$ are found to generate the corrected FT. This second example shows that the profile from the corrected FT coincides with that from the TMM, as shown in Fig. 3(b). The two simulation results preliminarily 
demonstrate the accuracy of the proposed correction to the FT formula, which presents the desired wavelength correctly from a periodic grating.

\section{CORRECTION OF THE FOURIER TRANSFORM FOR APERIODIC LATTICES WITH A HIGH -CONTRAST REFRACTIVE INDEX}

When considering an aperiodic grating design, the FT is the principal and rapid algorithm for generating aperiodic sequences of lattices from a target spectrum. The simulated annealing (SA) optimization algorithm is one of the design theories developed based on the FT [7]. Fig. 4(a) shows an aperiodic structure designed by applying an SA algorithm. Each vertical line in the structure corresponds to a narrow, low refractive index slot. $\Lambda$ is the minimum slot separation. All parameters of this aperiodic lattice (AL) are the same as that in Fig. 3(a) except that certain periodic sequences are broken by defects in order to obtain aperiodicity. The spectrum of this AL is displayed in Fig. 4(b), where we observe two separate profiles: TMM (blue line) and FT (red dotted line). The separation profiles of the blue and red dotted lines reveal the inaccuracy of the original FT for strongly scattering aperiodic gratings. 


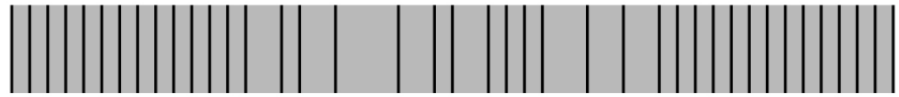

$1(\mathrm{x} 13) / 2 / 1 / 2 / 3.5 / 2 / 1 / 2 / 1 / 1 / 1 / 2.5 / 2 / 2 / 1(\mathrm{x} 13) \wedge$

(a)

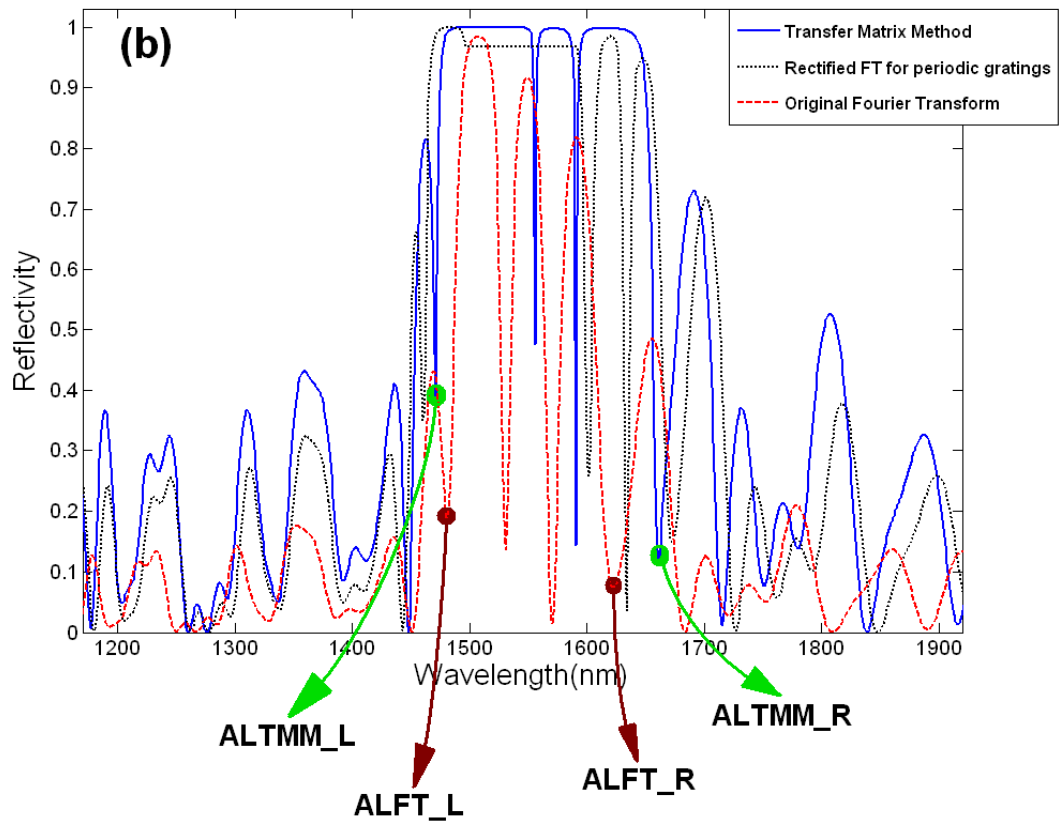

Fig.4. (a) An aperiodic design. Vertical lines represent narrow, low refractive index slots. (b)

The rough spectral correction in the black dotted line corresponds to the spectrum of the aperiodic grating, plotted using the corrected FT of periodic gratings

In this section, we further improve on the FT in order to accommodate aperiodic lattices (AL) under strong scattering conditions. First, the proposed rectified FT algorithms for correction of the periodic lattice in Fig. 3(a) is applied to the aperiodic grating, and the corresponding result is illustrated in black dotted line shown in Fig. 4(b). According to the spectral result, the wavelengths of the sub-bandwidths on both sides are nearly adjusted to the right wavelengths when comparing with the profile of the original FT. Modeling reveals a significant improvement of the sub-bandwidths on both sides in the spectral correction. For identical parameters other than aperiodic defects, using the corrected FT of this periodic grating to implement corrections to aperiodic gratings with aperiodic defects results in sub-bandwidths on both sides nearly identical to that of the accurate TMM, in particular regarding the location of the wavelength of each peak. In the next section, we investigate the corrections to the main 
bandwidth, where the corrected FT for periodic gratings is clearly inapplicable for aperiodic gratings.

\subsection{Principle of correction for aperiodic lattices}

In order to establish a corrected FT for aperiodic lattices, we first define the parameters as shown in Fig. 4. The left and right minimum extreme points on the TMM profile in the main band region are defined as $A L T M M_{-} L$ and $A L T M M_{-} R$. The definition of $A L F T_{-} L$ and $A L F T_{-} R$ are the left and right minimum extreme points of the original FT profile in the main band region. Thus, we obtain the proportion of wavelength adjustment main_adj:

main_adj $=\left(A L F T_{-} R-A L F T_{-} L\right) /\left(A L T M M_{-} R-A L T M M_{-} L\right)$.

Eq. (22) provides the required adjusted wavelength magnitude at each spectral point. We then add an additional formula towards the main band region for the improved FT for aperiodic lattices:

For $A L T M M_{-} L \leq \lambda \leq A L T M M_{-} R$,

$r\left(\lambda_{\text {main }}\right)=\tanh \mid-\frac{1}{4 \bar{n}^{2}} \int_{0}^{\infty} \frac{d \varepsilon(x)}{d x} \times$ Weightfactor $\times \exp \left(2 i\left(\frac{2 \pi}{\lambda_{\text {main }}^{a}}\right) \int_{0}^{x} n(z) d z\right) d x \mid$,

where $\lambda_{\text {main }}^{a}=\lambda_{\text {main }-1}^{a}+\left(\lambda_{\text {main }}-\lambda_{\text {main }-1}\right) \times$ main_adj $\left(\lambda_{\text {main }}\right)$.

The initial values of $\lambda_{\text {main }-1}$ and $\lambda_{\text {main }-1}^{a}$ are $A L T M M_{-} L$, and $\left(\lambda_{\text {main }}-\lambda_{\text {main }-1}\right)$ is the wavelength resolution distance.

The improved FT for strongly scattering aperiodic gratings is summarized as follows:

Find the periodic grating (PG) having an equivalent total length to the target aperiodic grating, and such that both periodic and aperiodic gratings have the same unit pitch length.

Calculate the corrected FT of the PG using Eqs. (16)-(21).

Implement the corrected FT of the PG to the initially corrected calculation of the target aperiodic grating.

(4) Calculate the corrected FT in the main band region using Eqs. (22)-(24) to establish the corrected FT of the AG, and implement it in order to adjust the inaccurate spectrum of the target aperiodic grating. 


\subsection{Simulation results}

Following the previous example of a periodic grating in Fig. 3(a), we now break some periodic sequences in order to create aperiodic structures, alter the total length, and perform simulations to verify the proposed corrected FT of aperiodic gratings. The unit pitch width, the two refractive indices with their occupied widths, the difference between the two refractive indices, and the central Bragg wavelength gratings remain the same as that in Fig. 3(a). The only variable is the period number that results in a certain total length.

The first example is an aperiodic grating with pitch distribution shown in Fig. 5(a.1). The total length of this aperiodic grating is $11.428 \mu \mathrm{m}$, the same as that in Fig. 4. The corresponding spectrum of this aperiodic grating, plotted using the corrected FT for aperiodic gratings, is shown in Fig. 5(a.2). In comparison with the profile from the original, inaccurate FT, our proposed correction for aperiodic gratings increases the accuracy of the spectrum. The peaks of the three modes located at accurate wavelengths in the main band region are the greatest improvement of our corrected FT of aperiodic gratings.

In the second example, we maintain the same total length as that in Fig. 5(a.1), but use different pitch distributions shown in Fig. 5(b.1). Fig. 5(b.2) displays its spectrum. The profile from the corrected FT for aperiodic gratings nearly matches that of the accurate profile from TMM, in particular for peaks of the four modes in the main band region located at accurate wavelengths.

In the third example, we reduce the total length of the aperiodic grating to $8.163 \mu \mathrm{m}$. The pitch distribution is shown in Fig. 5(c.1). The spectrum of this short aperiodic grating is illustrated in Fig. 5(c.2), where the two profiles from TMM and the corrected FT of aperiodic gratings almost match, successfully correcting the FT for aperiodic gratings. In the final example, we further reduce the length of the aperiodic grating shown in Fig. 5(d.1) to be $4.664 \mu \mathrm{m}$. A more accurate correction of the spectrum in terms of the corrected FT for aperiodic gratings is presented in Fig. 5(d.2). 

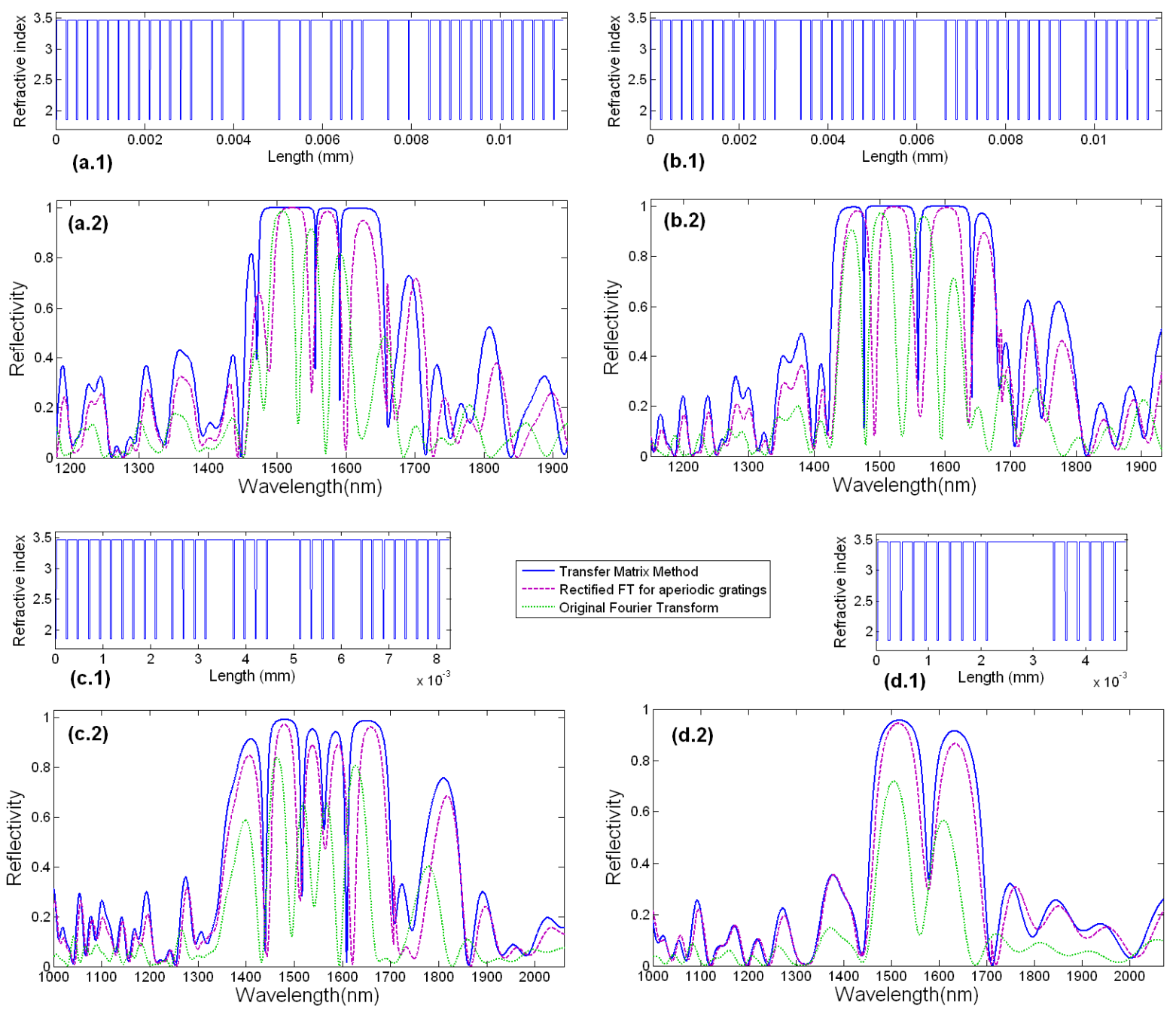

Fig.5. Several corrected outcomes using the aperiodic-specific corrected FT for strongly scattering aperiodic gratings with different lengths. Blue lines represent the accurate TMM as target profiles. Purple dash lines show the profiles from the corrected FT for aperiodic gratings. Green dotted lines are the profiles of the original, inaccurate FT

According to the four simulation examples, the rectified FT for aperiodic gratings greatly improves the original FT for high contrast refractive index grating conditions. Although the effect of the phase-accumulated error more or less accompanies an increase in total length, leading to a little incomplete correction of the bandwidth in the main band region as the total grating length increases, the proposed FT for aperiodic gratings still significantly reduces the phase-accumulated error and improves the spectrum accuracy of the original FT. In addition, modes in the main band region peak at accurate wavelengths. Furthermore, when applying a 
strongly scattering aperiodic grating in a resonator application, such as lasing, only extremely narrow bands survive inside the cavity, reducing the importance of bandwidth accuracy. Through our simulations, we demonstrated the ability to correct the FT of aperiodic gratings, particularly for short aperiodic gratings under strong scattering conditions.

\section{CONCLUSIONS}

We have developed a corrected Fourier transform (FT) formula for strongly scattering periodic and aperiodic lattices. The original FT, formed from the nonlinear Riccati differential equation, suffers from an inaccurate spectrum under strongly scattering conditions. We used an accurate reflective spectrum calculated using the transfer matrix method (TMM) as the target spectral profile in order to inversely obtain corrected parameters for the original FT formula. In this way, the original FT formula is largely corrected, as is the inverse FT formula. In contrast to assuming photon resonant behavior for the refractive index and coupling coefficient through models such as the Debye-Waller approximation, our approach involves inversely obtaining corrected wave numbers and the adjusted reflection intensity "weighting factor" from the accurate TMM for our corrected FT. In this way, we avoid directly assuming Bragg resonant behavior which may be more unpredictable and complicated than Debye-Waller approximation model in terms of aperiodic gratings. Simulations reveal that the phase-accumulation errors are hugely minimized, and the peak of each main mode is adjusted to the correct wavelength location. Finally, we believe that the proposed correction to the FT of strongly scattering lattices is helpful for algorithm development of aperiodic lattice design under high-contrast refractive index conditions so as to create a fast Fourier transform relationship between the aperiodic pitch distribution and the corresponding reflective spectrum.

\section{ACKNOWLEDGEMENTS}

The author thanks Dr. Subhasish Chakraborty for the author's $\mathrm{PhD}$ research experience in the laboratory of QCL-based Nanophotonics in School of Electrical and Electronic Engineering at the University of Manchester in United Kingdom. The author was a PhD student since 2010 
and she failed the proceeding of her PhD program in 2012. The research was finished in 2015.

\section{REFERENCES}

[1] Lazaro, J. A., Wessel, R., Koppenborg, J., Dudziak, G., Blewett, I. J., IEEE Photonic Tech. L. 2003, 15(1), 93-95, doi: 10.1109/LPT.2002.805809

[2] Verbist, M., Thourhout, D. V., Bogaerts. W., Opt. Lett. 2013, 38(3), 386-388, doi: 10.1364/OL.38.000386

[3] Jaggard, D. L., Kim, Y., J. Opt. Soc. Am. A Opt. Image Sci. Vis. 1985, 2(11), 1922-1930, doi: 10.1364/JOSAA.2.001922

[4] Zarifi, D., Soleimani, M., Abdolali, A., Phys. Rev. E 2014, 90(5), 053203, doi: 10.1103/PhysRevE.90.053203

[5] Bauer, C., Giessen, H., J. Optics 2014, 16(11), 114001, doi: $10.1088 / 2040-8978 / 16 / 11 / 114001$

[6]Janssen, T., Janner, A., Acta Cryst. B 2014, B70, 617-651, doi: $10.1107 / \mathrm{S} 2052520614014917$

[7] Chakraborty, S., Parker, M. C., Mears, R. J., Photonics Nanostruct. 2005, 3(2-3), 139-147, doi: 10.1016/j.photonics.2005.09.011

[8] Marshall, O. P., Chakraborty, S., Khairuzzaman, M., Folland, T., Gholinia, A., J. Appl. Phys. 2013, 113(20), 203103, doi: 10.1063/1.4807636

[9] Parker, M. C., Mears, R. J., Walker, S. D., J. Opt. A: Pure Appl. Opt. 2001, 3(6), S171-S183, doi: 10.1088/1464-4258/3/6/368

[10] Parker, M. C., Walker, S. D., IEEE Photonic Tech. L. 2002, 14(9), 1321-1323, doi: 10.1109/LPT.2002.801047

[11] Mikhnev, V. A., Vainikainen, P., IEEE T. Antenn. Propag. 2000, 48(2), 293-298, doi: $10.1109 / 8.833079$

[12] Kumar, A., Akhtar, M. J., IEEE Antennas and Propagation Society International Symposium 2013, 360-361, doi: 10.1109/APS.2013.6710841

[13] Bland-Hawthorn, J., Buryak, A., Kolossovski, K., J. Opt. Soc. Am. A Opt. Image Sci. Vis. 2008, 25(1), 153-158, doi: 10.1364/JOSAA.25.000153 
[14] Blanchard, R., Menzel, S., Pflugl, C., Diehl, L., Wang, C., Huang, Y., Ryou, J.-H., Dupuis, R. D., Negro, L. D., Capasso, F., New J. Phys. 2011, 13(11), 113023, doi: $10.1088 / 1367-2630 / 13 / 11 / 113023$

[15] Sarangan, A. M. 2007. Integrated Optics EOP-604. Chapter 4, 57-58.

\section{How to cite this article:}

Hsin Ying-Fei. Inverse correction of Fourier transforms for one-dimensional strongly scattering aperiodic lattices. J. Fundam. Appl. Sci., 2016, 8(2), 584-599. 